\title{
The Geometry of the Magnetosphere II : A Weakly Connected Model of the Geomagnetic Tail
}

\author{
R.C. WENTWORTH \\ Space Sciences Laboratory and Department of Mineral Technology \\ University of California, Berkeley, California 94720
}

(Received July 2, 1968)

\section{Introduction}

It was suggested in Part I (Wentworth, 1967) that the magnetosphere was pressed by the solar wind into three topologically distinct regions, the topologically un-connected upper and lower pole cap-tail flux tubes, and the central, connected core. In order to illustrate this process a model field configuration from two anti-parallel solenoids was discussed. It was suggested that two possible topologically distinct solutions existed, an un-connected solution in a strong, streaming plasma, and a connected solution in a weak, streaming plasma. It was implied that in the unconnected solution the boundaries of the upper and lower flux tubes approached each other asymptotically in the downstream direction.

However, this reasoning leaves out the finite boundary layer thickness. The question of what happens when the pure mathematical solution approaches closer than the boundary layer thickness was not considered. However, it is now felt that the result will be a fundamental change in the topology of the solution, that in fact the tail will be weakly connected across the region of contact as illustrated in Fig. la.

This possibility was suggested by $\mathrm{H}$. Alfven (private communication), who pointed out that electric fields across the neutral sheet would allow plasma to slip downstream through the weak connecting field without blowing it away, or being turned aside.

\section{Weakly Connected Flux Tubes}

There are two sources of the required electric field. First, multiple reflection of plasma from the upper and lower converging flux tubes just ahead of the area of contact (cf. Fig. 1a and 2a) will systematically polarize the plasma, building up an electric field across the equatorial plane. It is easy to see that reflection from both the upper and lower flux tubes will deflect protons out of the plane of the figure, and electrons in the opposite direction. Thus particles in the equatorial plane directed toward the area of contact can enter the neutral sheet under the simultaneous influence of this cross-electric field and the perpendicular magnetic field connecting the two flux tubes across the neutral sheet.

Once in the neutral sheet protons are deflected to the right (cf. Fig. 1b) and electrons to the left by the perpendicular northward magnetic crossing field. The resultant space charge distribution gives the required electric field. If $v_{s}$ is the velocity of the streaming plasma, 
a)

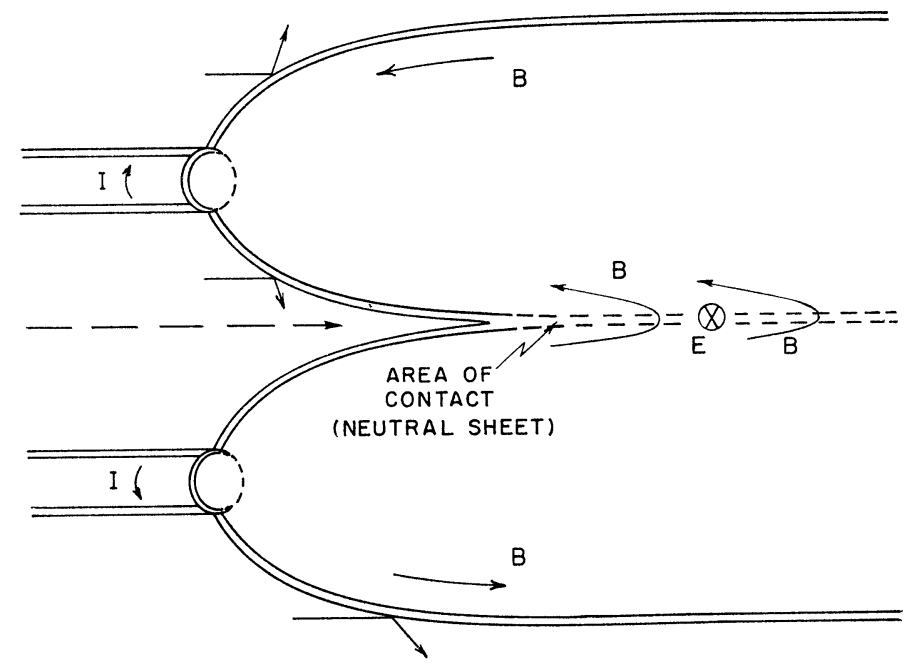

b)
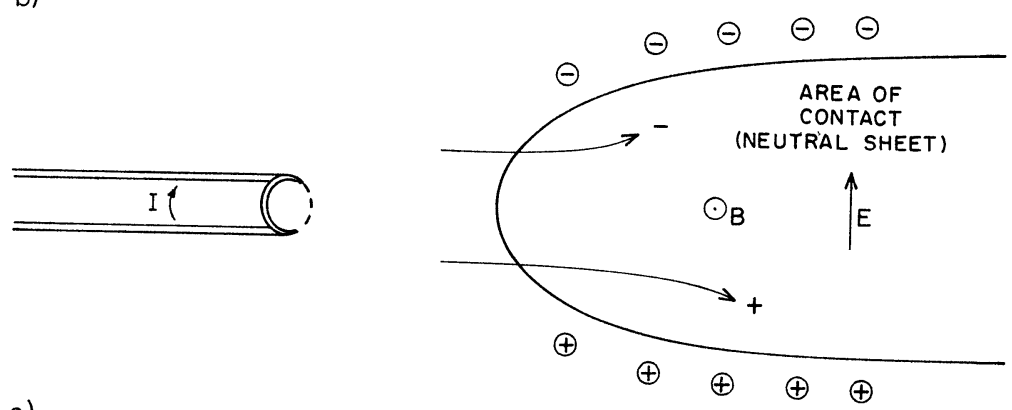

c)

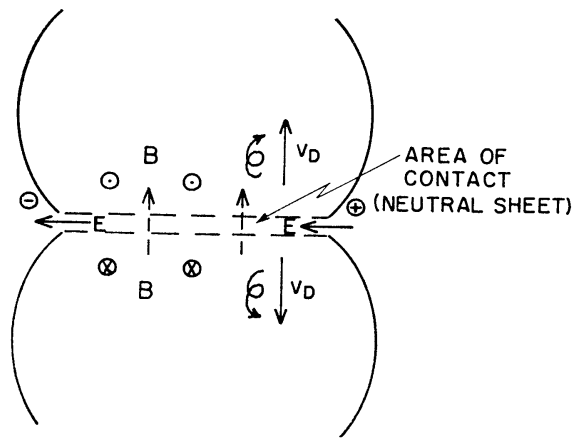

Fig. 1 a) Oppositely directed flux tubes from anti-parallel semiinfinite solenoids. The magnetic field is confined within a cavity bounded above and below (as well as on the sides away from the neutral sheet) by the normal pressure of specularly reflected plasma. The plasma streams through the nentral sheet under the simultaneous influence of the $v \times B$ and $E$ terms in the force expression.

b) Equatorial plane (or area of contact) viewed from above for the system of Fig. la. The separation of positive and negative charges by the perpendicular magnetic field produces the space charge along the boundary which gives rise to the electric field necessary to allow the plasma to slip through the magnetic field.

c) View looking down the tail in the equatorial plane of the system of Fig. 1a and 1b. The electric field across the tail drives plasma drifting into the interior from the neutral sheet, as described in the text. 
and $B_{p}$ the perpendicular component of the magnetic field connecting the two flux tubes across the region of contact, then the corresponding electric field is,

$$
E_{r s}=-v_{s} \times B_{p}
$$

\section{Weakly Connected Geomagnetic Tail}

The geometry of the geomagnetic tail is similar in important aspects to that of the antiparallel solenoids model just discussed. The main difference is that starting with the open, split-tail solution of Part I plasma enters the region between the converging upper and lower flux tubes of the tail from the flanks rather than head-on. Aside from that the situation is the same. Multiple reflection of plasma from the upper and lower flux tubes polarizes the plasma, setting up an electric field in the equatorial plane from the dusk to the dawn side. Thus plasma directed at the area of contact (cf. Figure 2a) can enter the neutral sheet under the simultaneous influence of this electric field and the perpendicular crossing magnetic field linking the two flux tubes. Finally, solar wind plasma flowing down the neutral sheet will be polarized, setting up the required electric field in the tail from the dusk boundary to the dawn boundary, as in Figure $2 \mathrm{~b}$.

\section{Plasma Sheet}

The same electric field will drive plasma drifting up and down away from the neutral

a)

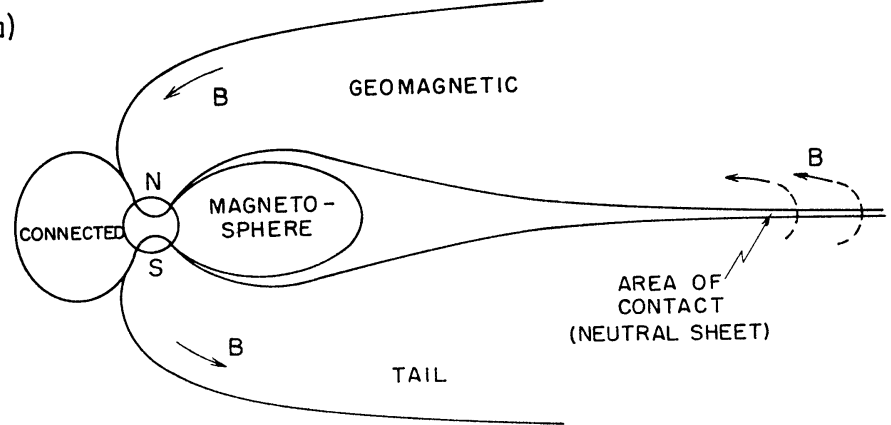

b)
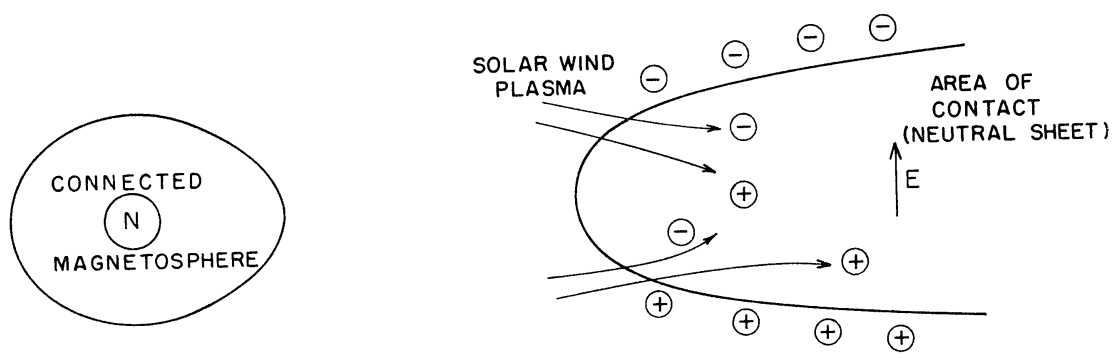

Fig. 2 a) The 3-component magnetosphere suggested by Wentworth (1967) with a weak connection of the upper and lower flux tubes across the neutral sheet.

b) Polarization of solar wind plasma flowing into the neutral sheet from the flanks as viewed from north of the equatorial plane. 
sheet into the tail proper, as shown in Figure 1c. The fact that this can happen follows from considering the inverse situation, namely plasma in the interior and a reversal of the electric field which will drive the plasma toward the boundary. The only thing that can happen when the particle reaches the boundary is that it crosses the boundary and escapes to the field-free region. However, since the orbit of the particle in this reversed situation is a mirror image of the orbit of the particle in the direct situation, it follows that a particle from the field-free region can penetrate into the interior. It is suggested that this is the source of the plasma sheet around the neutral sheet observed by Bame et al. (1967).

It is useful to point out at this time that other mechanisms have been suggested which allow plasma exterior to a magnetic field-domainated region to pass across the boundary and enter the interior. First, Piddington (1960) suggested that instabilities of an electrostatic nature exist in the boundary sheath of the magnetosphere, and that these instabilities could scatter ions into the stable trapping region. This suggestion was subsequently amplified by Bernstein et al. (1964), Fredericks et al. (1965), and Scarf et al. (1965). Second, it was suggested by Fejer (1965), and Wentworth (1965) that if the componet of the gradient of the interior magnetic field parallel to the boundary was finite then exterior particles could drift across the boundary and on into the interior. In this picture the particles would systematically drift into the interior, while in the first picture they would diffuse into the interior. The present suggestion represents a third possible mechanism whereby plasma outside a magnetic field can cross the boundary into the interior.

Finally, we should ' comment that specifics of the 'measurements of Bame et al. (1967) can not be applied directly to the question of the plasma sheet since they were made too

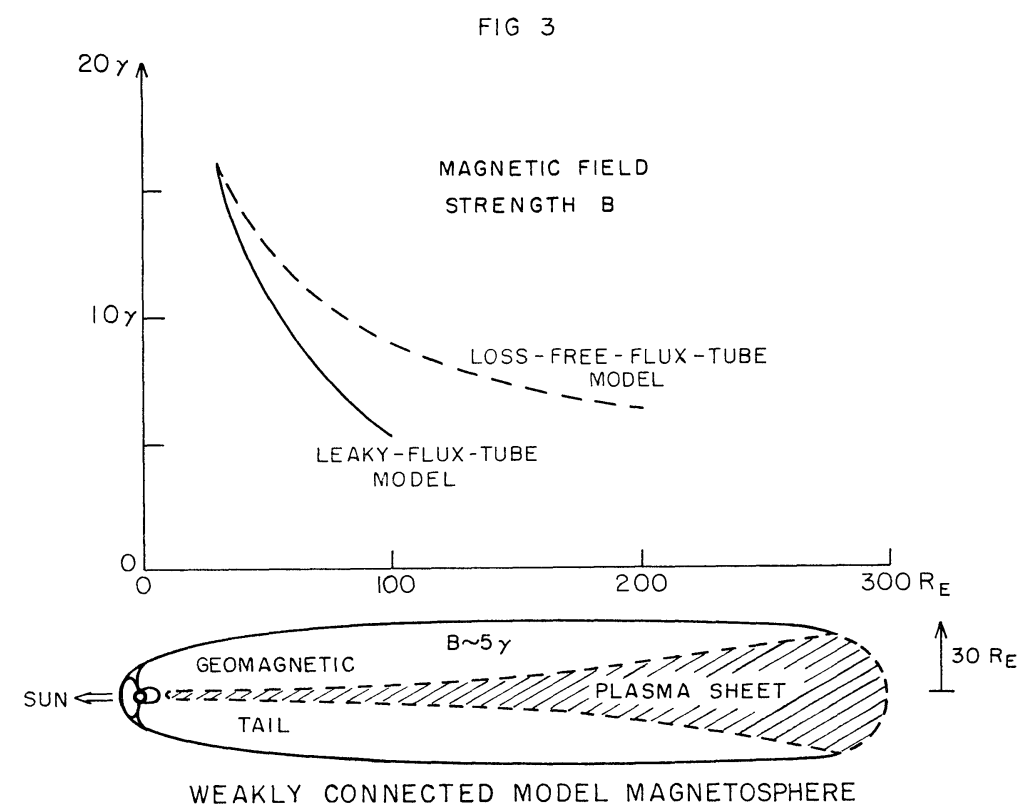

Fig. 3 Magnetic field strength and geometry of the geomagnetic tail for the weakly-connected model magnetosphere. 
close to the wake of the inner, closed magnetosphere, and not in the freely flowing downstream tail. This applies to the question of the energy spectrum of the plasma in the plasma sheet, which is radically different from that in the magnetosheath, and also to the question of whether the plasma sheet should fill the entire tail. It is possible, as later suggested, that the plasma sheet actually does flare out to fill the entire distant, downstream tail (cf. Fig. 3).

\section{Downstream Tail}

The expansion of the flux tube from a single solenoid in a streaming plasma was discussed in Part I. It follows from the conservation of magnetic flux that the magnetic field strength falls off in the manner depicted in the loss-free model of Fig. 3. However, in our present model flux is lost across the neutral sheet, and a reasonable estimation of this loss, in which the component of magnetic field across the neutral sheet is 25 percent of the main component paralled to the tail, is given in the leaky-flux-tube model, also in Fig. 3.

However, the tail cannot continue to expand beyond the point where the interior field along the boundary is less than the interplanetary field, around 5 gammas. This happens at about $-100 R_{E}$ downstream, where the diameter of the tail is on the order of $50-60 R_{E}$. Beyond this point it is suggested that the tail becomes increasingly inflated by interior plasma which provides the force necessary to maintain the tail against the external pressure.

Finally, if the component of magnetic field across the equatorial plane remains at approximately 1 gamma, then the entire magnetic flux in the tail will have been reconnected by about $-300 R_{E}$ downstream, as sketched in Fig. 3.

\section{Experimental Verification of the Weakly Connected Model}

An experimental verification of the essential features of the weakly connected model is a relatively simple matter for a plasma-gun experiment. The mathematical solution for an anti-parallel solenoids situation, as depicted in Fig. 1a, could be worked out by computer computations, is which the connections across the area of contact were ignored. Then the plasma-gun experiment could measure the actual topology of the resultant magnetic fields, map the region of contact containing perpendicular magnetic fields, and compare this region of contact with the result of the mathematical analysis.

\section{Discussion}

It has been suggested in Part I that the primary force responsible for the open, split-tail was normal plasma pressure of solar wind plasma along the boundary rather than more esoteric forces, such as viscous drag of various origins, or internal plasma or hydromagnetic wave pressure. It is still felt that this is the case, and that refinements of this model, such as that suggested in the present paper can account for the various effects still as yet unexplained. It is concluded:

i) Solar wind plasma in the neutral sheet is flowing downstream away from the earth. The suggestion that the plasma in the neutral sheet would blow a distant neutral point away downstream was first made by Dessler (1964), and the present view is in accord with 
this suggestion.

ii) This downstream-flowing plasma is electrically polarized by the perpendicular component of magnetic field northward across the neutral sheet, the resultant space charge giving rise to a dusk-to-dawn electric field across the tail. This cross electric field allows the plasma to slip downstream through the perpendicular magnetic field component without blowing it downstream.

iii) The cross electric field causes plasma to drift from the region of contact in the neutral sheet up and down into the tail proper, thus being responsible for the plasma sheet observed by Bame et al. (1967).

\section{Acknowledgements}

I wish to acknowledge a stimulating discussion on this subject with H. Alfven. Many of these ideas were developed during a recent Guggenheim. Fellowship in 1966-67. This research was supported dy NASA Grant NsG 243.

\section{References}

Axford, W.I., H.E. Petschek, and G.L. Siscoe, Tail of the magnetosphere, J. Geophys. Res., 70, 1231-1236, 1965.

Bame, S.J., J.R. Asbridge, H.E. Felthauser, E.W. Hones, and I.B. Strong, Characteristics of the plasma sheet in the earth's magneto-tail, J. Geophys. Res., 72, 113-129, 1967.

Bernstein, W., R.W. Fredericks, and F.L. Scarf, A model for a broad disordered transition between the solar wind and the magnetosphere, J. Geophys. Res., 69, 1201-1210, 1964.

Dessler, A.J., Length of magnetospheric tail, J. Geophys. Res., 69, 3913-3918, 1964.

Fejer, J.A., Geometry of the magnetospheric tail and auroral current systems. J. Geophys. Res., 70, 49724975, 1965.

Fredericks, R.W., F.L. Scarf, and W. Bernstein, Numerical estimates of superthermal electron production by ion acoustic Waves in the transition region, J. Geophys. Res., 70, 21-28, 1965.

Piddington, J.H., Geomagnetic storm theory, J. Geophys. Res., 65, 93-106, 1960.

Scarf, F.L., W. Bernstein, and R.W. Fredericks, Electron acceleration and plasma instabilities in the transition region, J. Geophys. Res., 70, 9-20, 1965.

Wentworth, R.C., Diamagnetic ring current theory of the neutral sheet and its effects on the topology of the anti-solar magnetosphere, Phys. Rev. Letters, 14, 1008-1011, 1965.

Wentworth, R.C., The geometry of the magnetosphere, J. Geophys. Res., 72, 4582-4586, 1967. 